\title{
Qualitative prediction of blood-brain barrier permeability on a large and refined dataset
}

\author{
Markus Muehlbacher • Gudrun M. Spitzer • \\ Klaus R. Liedl · Johannes Kornhuber
}

Received: 19 June 2011/Accepted: 10 October 2011/Published online: 23 November 2011

(C) The Author(s) 2011. This article is published with open access at Springerlink.com

\begin{abstract}
The prediction of blood-brain barrier permeation is vitally important for the optimization of drugs targeting the central nervous system as well as for avoiding side effects of peripheral drugs. Following a previously proposed model on blood-brain barrier penetration, we calculated the cross-sectional area perpendicular to the amphiphilic axis. We obtained a high correlation between calculated and experimental cross-sectional area $(\mathrm{r}=0.898, \mathrm{n}=32)$. Based on these results, we examined a correlation of the calculated cross-sectional area with blood-brain barrier penetration given by $\log B \mathrm{~B}$ values. We combined various literature data sets to form a large-scale $\log B B$ dataset with 362 experimental $\log B B$ values. Quantitative models were calculated using bootstrap validated multiple linear regression. Qualitative models were built by a bootstrapped random forest algorithm. Both methods found similar descriptors such as polar surface area, $\mathrm{pKa}, \log P$, charges and number of positive ionisable groups to be predictive for $\operatorname{logBB}$. In contrast to our initial assumption, we were not able to obtain models with the cross-sectional area chosen as relevant parameter for both approaches. Comparing those two different techniques, qualitative random forest models are better suited for blood-brain barrier permeability
\end{abstract}

Electronic supplementary material The online version of this article (doi:10.1007/s10822-011-9478-1) contains supplementary material, which is available to authorized users.

M. Muehlbacher · J. Kornhuber $(\bowtie)$

Department of Psychiatry and Psychotherapy,

Friedrich-Alexander University of Erlangen-Nuremberg,

Erlangen-Nuremberg, Germany

e-mail: johannes.kornhuber@uk-erlangen.de

G. M. Spitzer · K. R. Liedl

Theoretical Chemistry, Center for Molecular Biosciences,

University of Innsbruck, Innsbruck, Austria prediction, especially when reducing the number of descriptors and using a large dataset. A random forest prediction system $\left(\mathrm{n}_{\text {trees }}=5\right)$ based on only four descriptors yields a validated accuracy of $88 \%$.

Keywords Blood-brain barrier · Central nervous system · Membrane permeability $\cdot$ QSAR $\cdot \operatorname{LogBB} \cdot$ Random forest
Abbreviations
BBB Blood-brain barrier
CNS Central nervous system
CSA Cross-sectional area
$\operatorname{LogBB}$ Logarithmic ratio between concentration of a compound in brain and blood
P-Gp P-glycoprotein transporter
TPSA Topological polar surface area

\section{Introduction}

The blood-brain barrier (BBB) is a complex system, tightly regulating the transport from and to the central nervous system (CNS) [1]. It separates the systemic bloodstream from the CNS and is therefore important for drug diffusion and transport between them [2]. Drugs targeting the CNS need to be able to pass the BBB to reach their target [3]. In contrast, low BBB permeability reduces the chance of undesirable CNS-related side effects $[4,5]$. Therefore an early estimation of BBB permeability would be highly valuable for drug design [6,7]. The relevance of BBB permeability of therapeutic drugs has been reported in the context of numerous clinical dysfunctions, like dementia [8] and other clinical disorders [9-11].

The most common numeric value describing permeability across $\mathrm{BBB}$ is the $\log \mathrm{BB}$ [12]. It is defined as 
ogarithmic ratio between the concentration of a compound in brain and blood (Eq. 1).

$\log \mathrm{BB}=\log \left(\frac{c_{\text {Brain }}}{c_{\text {Blood }}}\right)$

Unfortunately, experiments to measure $\log B \mathrm{~B}$ are timeconsuming, laborious and expensive in vitro [13-16] and even more in vivo $[17,18]$. So it is not surprising that the number of published experimental values is limited. Experimental methods to assess BBB permeability range from artificial membranes and complex cell culture systems to in vivo methods. The PAMPA assay uses artificial membranes to observe passive (effective) membrane permeability, quantified by $\mathrm{P}_{\text {eff }}[19,20]$. Obviously, those experiments are only able to observe permeability, neglecting the special characteristics of the BBB. Nevertheless, results from these studies support the validity of lipid bilayer systems as strongly simplified representations of the BBB. The main drawback of cell-free methods is that they neglect active transporters acting at the BBB and therefore incorrectly predict substrates to transport systems [21]. Numerous active transport systems and efflux transport systems play an important role at the BBB $[22,23]$. One of the most commonly reported transport systems acting at the BBB is P-Glycoprotein (P-Gp) [2426]. In contrast, in vivo methods, like in situ brain perfusion [17], are able to capture real BBB permeability as given by PS (permeability surface product) or $\log P S$ values [27].

Due to these experimental difficulties, it is not surprising, that BBB is frequently addressed via computational approaches. Computer-aided methods applied to this field of interest include multiple linear regression [28-32], bagged regression [33], partial least square analysis [3437], support vector machines [38-40] and artificial neural networks [39, 41]. These methods are frequently combined with descriptor selection algorithms based on genetic algorithms to name only one [42, 43]. A comprehensive overview of previous models for BBB prediction has been published by Vastag and Keseru [44].

Depending on the size of the dataset, the number of descriptors, and the mathematical approach for prediction range from rough guidelines to quantitative predictions. Complex methods like partial least square analysis and artificial neural networks suffer from the drawback of being hard to interpret, whereas simple methods like multiple linear regression often yield less accurate results or even only rough guidelines [45]. Although different mathematical techniques make it hard to compare the results directly, the performance decreases strongly with larger datasets. High squared correlation coefficients above 0.85 are reported frequently for focused data sets with a size of approximately 50-90 compounds [31]. Predictions based on larger compound collections with a size of over 200 compounds resulted mainly in "rules of thumb" for good BBB permeability [45]. Altogether, these findings clearly show that there is still need for further research on BBB permeability [46].

Summarizing recent work, there is broad agreement on the importance of some molecular properties and descriptors which have been found in numerous publications to influence BBB permeability [45]:

- The descriptor most frequently reported with BBB permeability is the polar surface area. The majority of publications report correlation of $\log \mathrm{BB}$ with the polar surface $[30,47]$ or a property closely related to it [35]. The sum of oxygen and nitrogen atoms for example is extremely cheap in computation-time, but has still proven to be useful.

- There is consensus that BBB permeability is also highly influenced by lipophilicity $[48,49]$. One way to quantify lipophilicity is $\log P$, the logarithmic partition coefficient between 1-octanol and water. However, the ability of $\log P$ to represent lipophilicity come under discussion recently [50], as octanol is a good hydrogen donor and therefore probably not a typical apolar solvent, even more when used as a calculated in silico descriptor [50, 51]. In addition to that, $\log P$ is defined for the neutralized state of a compound. $\log P$ values for ionized (e.g. protonated) compounds are basically not defined [52]. Liu et al. [47] introduced 'lipoaffinity' as an easily-accessible descriptor. It is calculated by adding the contributions to the $\log P$ values of all but nitrogen and oxygen atoms.

- Molecular flexibility has also been reported to influence BBB permeability. This is in agreement with the theory we used in this study (see below), since rigid molecules seem to fit less well to the membrane than more flexible ones (given that both molecules have approximately the same weight) [53]. A simple descriptor representing molecular flexibility would be the number of rotatable bonds, for example [29].

In this study we followed an approach based on physicochemical properties to address permeation across the BBB, proposed by Fischer et al. [53]. According to this hypothesis, the process of integrating a compound into a membrane can be split into essential steps that can be added up to form the process of membrane permeation:

- In the first step the compound needs to be desolvated from the aqueous environment. The process of desolvation is often addressed by molecular dynamics simulations [54]. Simultaneously, a cavity, appropriate for embedding the compound within the membrane is created. The amount of energy required to create this cavity is correlated to the energy needed to insert a molecule into the membrane. Fischer et al. [53] assume that the size of this cavity is crucial for membrane permeation. 
- In the second step the compound is inserted into the cavity. It is stabilized by electrostatic interactions with the polar headgroup of lipids and hydrophobic interactions with the core region of the lipid bilayer [55].

- Finally, the compound needs to resolvate behind the lipid bilayer. This process is similar to the reversion of the solvation process.

Figure 1 schematically illustrates how a molecule is inserted into a membrane according to this hypothesis. Based on this theory Gerebtzoff and Seelig [56] introduced the cross-sectional area (CSA) of a molecule as a novel descriptor to presumably represent BBB permeability. Because of its well-founded physico-chemical background it promised to achieve good predictability and interpretability, although this descriptor neglects all thermodynamic aspects of desolvation and resolvation. Descriptors based on valid mechanistic models have proven to contribute to the design and optimization of drug molecules [57]. Thus we reproduced this promising molecular descriptor and critically analysed its ability to predict BBB permeability. For this purpose, we compiled a large data set with experimental $\log B \mathrm{~B}$ values from numerous published datasets, instead of using single focused sets.

\section{Methods}

\section{Calculation of the CSA}

We calculated the amphiphilic axis and CSA, as described in detail in Gerebtzoff and Seelig [56]. Modifications were

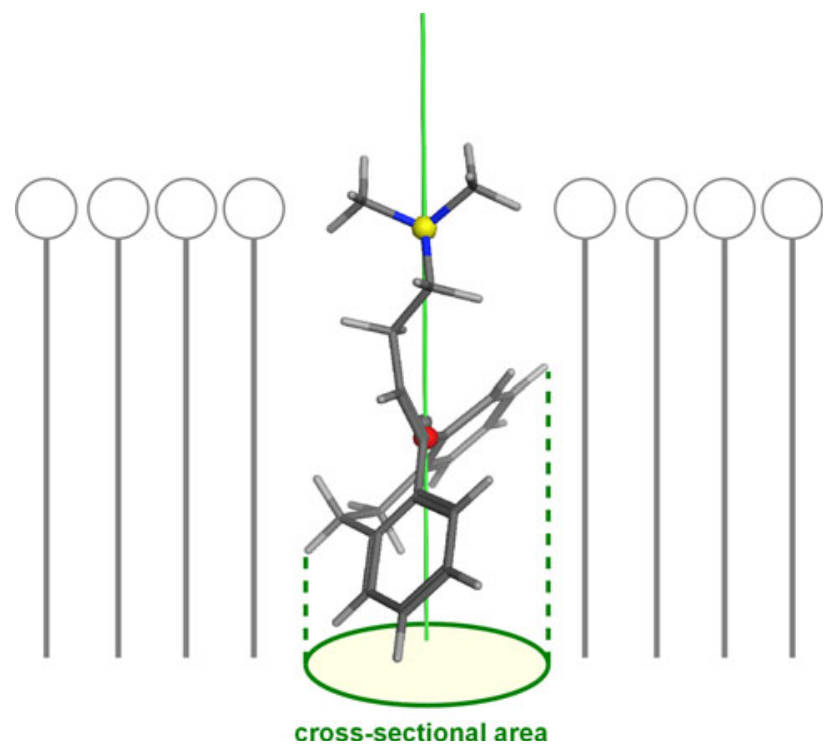

Fig. 1 The cross-sectional area (CSA) has been introduced as a measure for the area occupied by a compound after insertion into a lipid membrane. Local polarity of the membrane determines the orientation of the ligand introduced wherever the description was not clear or the results did not match our expectations. The following section describes and explains these modifications.

The amphiphilic axis is defined by the hydrophobic and hydrophilic center of a molecule. The hydrophilic center was calculated by averaging oxygen and nitrogen atom positions weighted by their contribution to the topological polar surface area (TPSA). Assuming that hydrogen bonds mainly influence BBB permeability [32], we decided to consider only nitrogen and oxygen as hydrophilic atoms and neglect sulphur atoms. The weighting factors were based on TPSA provided by MOE [58]. To emphasize the increased polar character of charged atoms compared to polarized atoms, we assigned a factor of 100 to charged atoms according to Eq. 2, where wf is the weighting factor, $\mathrm{z}$ is the charge and $\mathrm{w}_{0}$ is the weighting factor according to the TPSA.

$\mathrm{wf}=100 \cdot \mathrm{z}+\mathrm{w}_{0} \cdot(1-\mathrm{z})$

Halogen and carbon atoms were taken into account to place the hydrophobic center. Hydrophobic atom positions were weighted by their contribution to $\log P$ prediction by MOE $(\log P(\mathrm{o} / \mathrm{w}))$ [59]. This fragment-based calculated $\log P$ suggests that halogen atoms have a large negative contribution to $\log P$, which results in a displaced hydrophobic center for molecules containing halogen atoms. Thus we removed the logarithm before weighting to avoid negative contributions. Removal of the logarithm resulted in a more intuitive placement of the hydrophobic center (see Fig. 2.)

According to the mechanism outlined by Fischer et al. [53], a molecule inserts into a membrane along the amphiphilic axis. The CSA reflects the area occupied by the molecule when projected to the plane perpendicular to the amphiphilic axis (see Figs. 1, 3). Projecting a molecule onto an area reduces computational efforts from 3D into 2D space, which dramatically increases the calculation speed for larger molecules in contrast to the published procedure [56].

Calculation of amphiphilic axis and CSA were performed with MOE [60] using its scripting language SVL (complete script is available as supplementary information). Partial charges were calculated using MMFF94x forcefield. Protonation states were assigned according to physiological $\mathrm{pH}$ of 7.4.

\section{Experimental CSA values}

To validate our CSA calculations, we compared our results with experimental CSA values [56]. We obtained all structures as SDF files from PubChem [61]. The reported dataset [56] consists of 32 compounds with experimental CSA values for $\mathrm{pH} 7.4$ and 8 . The experimental CSA at $\mathrm{pH}$ 7.4 was used, as it represents physiological pH. Carebastine 
Fig. 2 Comparison of two different strategies to calculate the hydrophobic center (red sphere) for compounds with halogen atoms (like perphenazine). On the left side, the hydrophobic center is calculated weighting atom positions by their contribution to $\log P$ prediction; on the right side the calculation is done with modifications presented in this study

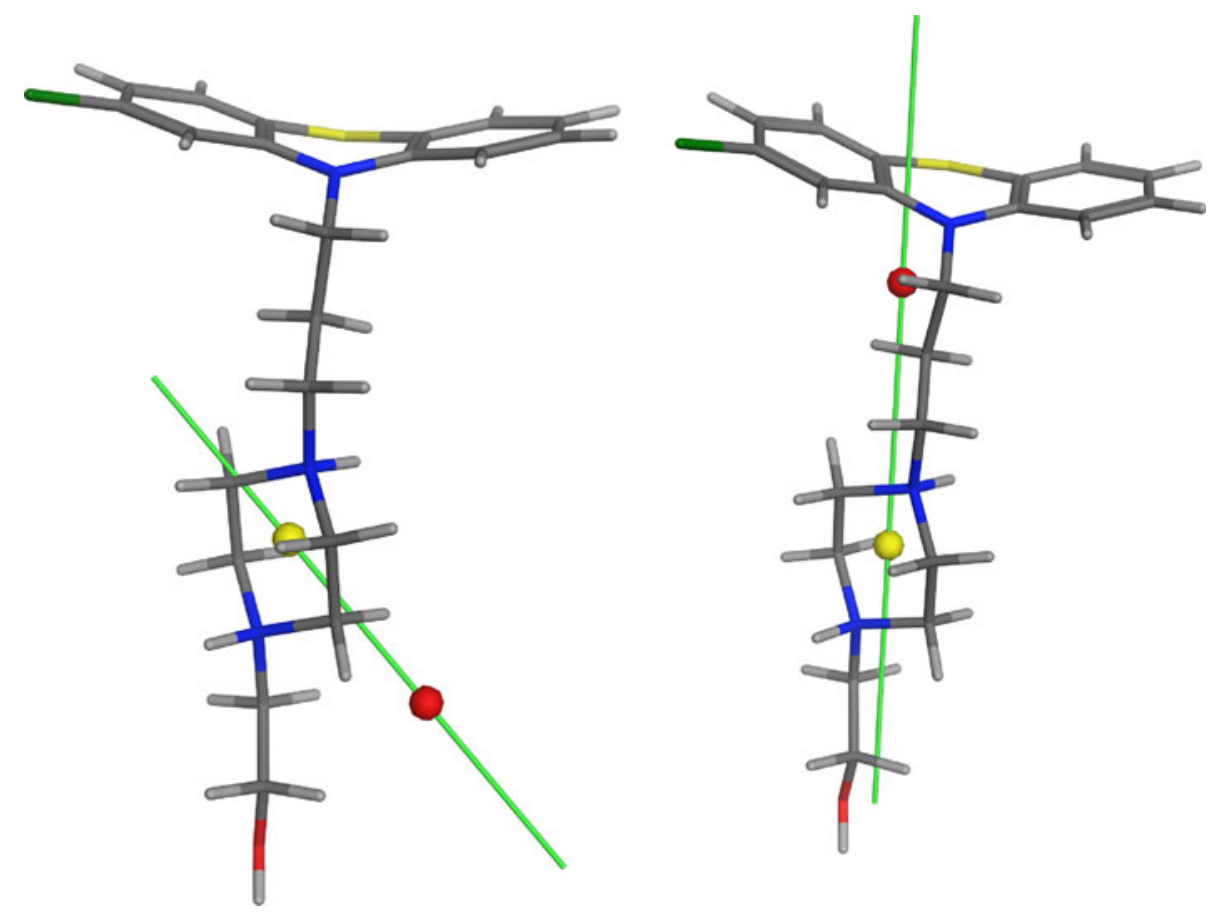

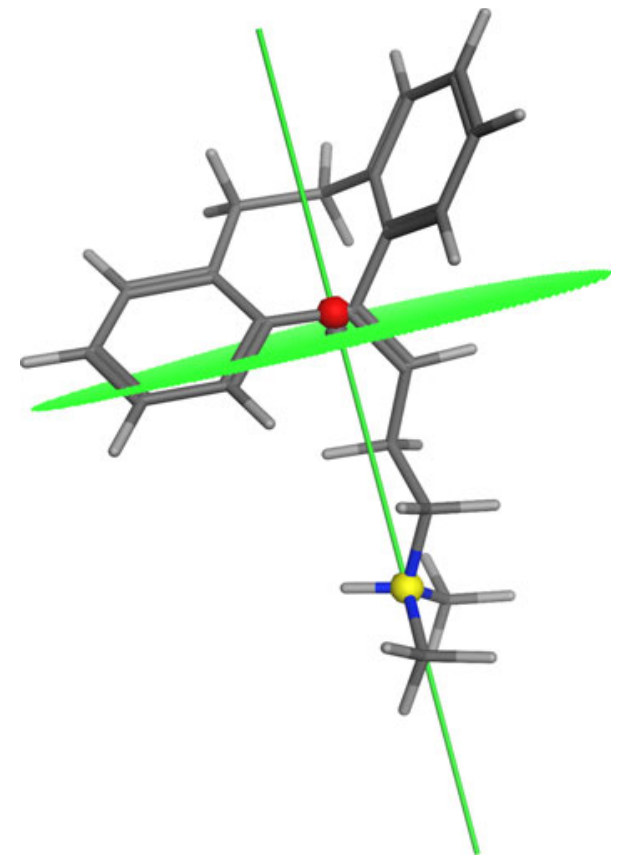

Fig. 3 Amitriptyline with hydrophilic center (yellow sphere), hydrophobic center (red sphere), amphiphilic axis (green line) and CSA (green dotted area). This BBB-permeable compound illustrates the role of the amphiphilic axis and the CSA

was excluded from the set since the two CSA values for $\mathrm{pH}$ 8 and 7.4 differed significantly. We also removed betacyclodextrin from the dataset, since it is not a typical druglike molecule with a molecular weight over $1,000 \mathrm{Da}$ (see Lipinski's rule-of-5 [62]). For each compounds the most stable conformation according to its conformational energy calculated by Omega (version 2.0) was used to calculate the CSA.

Experimental $\log B B$ values

In contrast to the small number of experimental CSA values, various studies containing experimental $\log B \mathrm{~B}$ values have been published. To investigate the ability of CSA for $\log B B$ prediction we combined all available published datasets and generated a novel large $\log B \mathrm{~B}$ dataset. Our dataset consisted of 195 compounds from Vilar et al. [63], 119 compounds from Platts et al. [64], 38 compounds from Naranayan and Gunturi [43], 94 compounds from Mente and Lombardo [33], 147 compounds from Zhang et al. [40], 197 compounds from Abraham et al. [65], 168 compounds from Garg and Verma [66], 106 compounds from Guerra et al. [67], 95 compounds from Rose et al. [68], 36 compounds from Kelder et al. [31], 165 compounds from Konovalov et al. [69] and 36 compounds from Zerara et al. [70]. Many compounds were reported multiple times with similar or identical $\operatorname{logBB}$ values, especially drugs with CNS-related effects such as antidepressants or neuroleptics. The average of the $\log B \mathrm{~B}$ values was used for identical compounds reported more than once. After removing duplicate structures, we ended up with 362 unique compounds with experimental $\log B \mathrm{~B}$ values ranging from -2.2 to +1.6 .199$ $\log B B$ values were positive, 163 were negative or zero.

From this set we also wanted to exclude actively transported compounds, since their mechanism of passing the BBB is different to those passively entering CNS. Therefore we searched for substrates of P-Gp, one of the 
major transport systems acting at the BBB, in three previously published datasets [71-73]. Combining results from these sources we excluded 18 known substrates of P-Gp (bunitrolol, cimetidine, digoxin, domperidone, etoposide, fexofenadine, flunitrazepam, levodopa, loperamide, methotrexate, morphine, nevirapine, phenytoin, quinidine, risperidone, triflupromazine, vincristine, yamatetan). In addition to these 18 compounds, six compounds (chlorpromazine, doxorubicin, nelfinavir, saquinavir, verapamil, vinblastine) are reported ambiguously in the publications, thus we did not exclude them.

To the best of our knowledge, this is to date the largest set of quantitative $\log B \mathrm{~B}$ values, compiled from various resources. This dataset promises to be a very elaborate and refined selection of compounds. The complete dataset can be found in the supplementary material.

\section{Descriptor calculation}

We calculated all descriptors provided by MOE 2010.10 [59] and all from ACD/Labs (version 10.0) [74], that could be calculated for all compounds. A complete list of descriptors used is included the supplementary information. In addition, we calculated descriptors reported to be useful in other publications addressing BBB permeability, as far as we were able to reproduce them. Table 1 lists all additional descriptors together with a reference to their original publication. We also implemented size intensive descriptors using molecular weight as a normalizing factor [75]. Finally, our data set comprised over 880 descriptors, ranging from simple atom counts to computationally intensive quantum-mechanical properties.

Quantitative models: beam search and multiple linear regression

A large number of potentially predictive descriptors prompted us to systematically reduce dimensionality (the number of descriptors) used to construct and validate the models. A beam search algorithm (width $=$ number of descriptors $=79$ ) was applied to preselect potentially predictive descriptors [78]. For each combination a bootstrapped multiple linear regression was calculated and the squared correlation coefficient was returned as fitness criterion. We limited the maximum number of generations and subsequently the number of descriptors simultaneously taken into account to 10 and selected the best multiple linear regression model per generation.

Qualitative models: beam search and random forest

To generate qualitative models our dataset was split into $\mathrm{BBB}$ permeable $(\log \mathrm{BB} \geq 0.3, \quad \mathrm{n}=126)$ and non- permeable $(\log \mathrm{BB} \leq-0.3, \mathrm{n}=76)$ compounds. The compounds between the two limits $(n=142)$ were excluded from the process, as they do not show strong characteristics of BBB permeable or non-permeable, respectively. These limits were adapted from Abraham et al. [65], who assume an experimental error of about $0.3 \log$ units $(\log B B$ values range from -2.2 to 1.6 ). We then performed a beam search from 1 to 5 descriptors (width $=$ number of descriptors $=72$ ). As qualitative model we constructed a random forest model for each combination $\left(n_{\text {tree }}=5\right.$, depth $=5$ ), validated by a bootstrapping procedure (sample ratio $=1.0$, number of validations $=100$ ). Accuracy was used as the main performance criteria. Again, we captured the best models per generation.

All models were calculated using RapidMiner (version 5.1.1) and the Weka's implementation of a random forest algorithm. Correlation coefficients were calculated according to Pearson.

\section{Results and discussion}

We calculated the CSA for 32 compounds and compared it with experimental values taken from Gerebztoff and Seelig [56]. Similar to the original work we also achieved a good correlation $(r=0.898)$ to experimental CSA values.

Quantitative models to predict $\log B B$

The main intention of the present study was to investigate a correlation between CSA and BBB permeability, as suggested by Gerebtzoff and Seelig [56]. We therefore constructed multiple linear regression models using a beam search algorithm for feature selection (up to 10 descriptors).

Table 2 shows the squared correlation to increase with respect to the number of descriptors. Simultaneously, the validated squared correlation is constant or even decreases for more than 5 descriptors. Overall, statistical parameters improve only slightly from 5 to 10 descriptors, although the number of descriptors used is doubled. Thus influence of additional descriptors must be questioned. The validated squared correlation increases constantly up to 5 descriptors. So we consider 5 as the maximum number of descriptors to avoid overfitting.

In agreement with previous studies, TPSA is highly important for BBB permeability. The number of polar atoms (n_pol) and a descriptor taken from Feher et al. [30] (I3), followed by the number of positive ionisable groups (n_PI) and a descriptor developed for this study (PDist) were also found to influence BBB permeability, as well as the number of hydrogen bond acceptors (a_acc).

In contrast to our expectations, CSA never appeared in the most predictive models. This leads to the question why 
Table 1 List of molecular descriptors developed or reproduced in addition to the standard descriptors by $\mathrm{ACD} /$ Labs 10.0 and MOE 2010.10

\begin{tabular}{|c|c|c|}
\hline Descriptor & Description & Reference \\
\hline AA & Length of the amphiphilic axis & {$[56]$} \\
\hline CSA & Cross-sectional area perpendicular to the amphiphilic axis & {$[56]$} \\
\hline LAA & Length along the amphiphilic axis & \\
\hline NOOM & Number of atoms above the hydrophilic center & \\
\hline VOOM & Volume above the hydrophilic center & \\
\hline li & Longest distance from an ionized atom to another atom & \\
\hline $\mathrm{mpc}$ & Longest distance from the atom with the highest partial charge & \\
\hline n_COOH & Number of carboxylic acid functions & {$[76]$} \\
\hline n_hal & Number of halogen atoms & \\
\hline n_ion & Number of ionized atoms & \\
\hline QMAXneg & Highest negative partial charge & [76] \\
\hline QMAXpos & Highest positive partial charge & [76] \\
\hline QSUMH & Sum of all partial charges on hydrogen atoms & \\
\hline QSUMO & Sum of all partial charges on oxygen atoms & {$[76]$} \\
\hline QSUMN & Sum of all partial charges on nitrogen atoms & \\
\hline n_OpN & Sum of nitrogens and oxygen atoms & {$[35]$} \\
\hline N_XpC & Sum of halogens and carbon atoms & \\
\hline LA & Lipoaffinity & [47] \\
\hline $\log P$-NO & $\log P$-number of oxygen and nitrogen atoms & {$[35]$} \\
\hline $\mathrm{I} 3$ & +1 for amines, -1 for acids, otherwise 0 & {$[30]$} \\
\hline n_PI & Number of positive ionizable groups & \\
\hline QMAXneg & Highest negative partial charge & [76] \\
\hline QMAXpos & Highest positive partial charge & [76] \\
\hline QSUMH & Sum of all partial charges on hydrogen atoms & \\
\hline QSUMO & Sum of all partial charges on oxygen atoms & [76] \\
\hline QMINN & Lowest partial charge on nitrogen atoms & \\
\hline QSUMN & Sum of all partial charges on nitrogen atoms & \\
\hline QMEANN & Average partial charge in nitrogen atoms & \\
\hline Qamines & Average partial charge on amines & \\
\hline LA & Lipoaffinity & {$[47]$} \\
\hline n_pol & Number of polar atoms & {$[77]$} \\
\hline n_amines & Number of amines & \\
\hline $\mathrm{n} \_\mathrm{pN}$ & Number of protonated nitrogen atoms at $\mathrm{pH} 7$ & \\
\hline
\end{tabular}

CSA does not contribute to BBB prediction as much as TPSA, for example. For BBB-permeable compounds Gerebtzoff and Seelig, [56] suggest that there is an upper limit for CSA at $80 \AA^{2}$. Figure 4 a shows a scatterplot of $\log \mathrm{BB}$ versus calculated CSA values for our large dataset, to further analyse this hypothesis. For 11 compounds, both experimental and calculated values for CSA and $\log B \mathrm{~B}$ were available. Overall, this plot does not show a clear correlation between CSA and $\log B$ B. As suggested in the original publication, we also investigated our dataset with respect to $\log \mathrm{D}$ (at $\mathrm{pH} 7.4$ ) and $\mathrm{CSA}$. In contrast to the original publication Fig. 4b shows no significant separation by $\log \mathrm{D}$ and CSA. A limit for BBB permeable compounds reflected by the CSA could not be determined.
Correlation between CSA and number of atoms

Searching for structural and chemical information covered by CSA, we tested its correlation with all other descriptors. Overall, various descriptors correlate remarkably well with the CSA. Table 3 lists the correlation with prominent other descriptors, including those from the models listed in Table 2. The majority of those are based on properties, easily obtainable from the structure. Remarkably, CSA is highly correlated to numerous simple descriptors that are easier to calculate, such as the number of atoms (see Fig. 5). A good correlation $(r=0.959)$ between those two properties suggest that CSA can be seen as derivative of the number of atoms. A high correlation of approximately 0.9 
Table 2 Squared correlation coefficients (raw and bootstrap validated) of the best models with 1-10 descriptors constructed with beam search using multiple linear regression and squared correlation as performance criterion

\begin{tabular}{|c|c|c|c|}
\hline $\mathrm{n}_{\mathrm{atts}}$ & Descriptor names & $\mathrm{r}^{2}$ & $\mathrm{r}_{\text {bootstrapping, } 100}^{2}$ \\
\hline 10 & $\begin{array}{l}\text { si_TotalFormalCharge, prot_n_pol, neutral_n_pol, a_don, PEOE_VSA_POL, PDist, I3, chi1, } \\
\text { logPow-logWeight, n_PI }\end{array}$ & 0.585 & 0.508 \\
\hline 9 & $\begin{array}{l}\text { si_TotalFormalCharge, prot_n_pol, neutral_n_pol, PEOE_VSA_PNEG, PDist, I3, apol, } \\
\text { logPow-logWeight, n_PI }\end{array}$ & 0.577 & 0.534 \\
\hline 8 & si_TotalFormalCharge, prot_n_pol, neutral_n_pol, a_don, PDist, chi1, logPow-logWeight, n_PI & 0.568 & 0.553 \\
\hline 7 & prot_n_pol, neutral_n_pol, a_don, PDist, chi1, logPow-logWeight, n_PI & 0.558 & 0.537 \\
\hline 6 & prot_n_pol, neutral_n_pol, a_acc, PDist, prot_logPow-logWeight, neutral_n_PI & 0.544 & 0.520 \\
\hline 5 & prot_n_pol, neutral_n_pol, a_acc, $\log P$ Pow-logWeight, n_PI & 0.533 & 0.521 \\
\hline 4 & TPSA, I3, logPow-logWeight, n_PI & 0.515 & 0.499 \\
\hline 3 & prot_n_pol, neutral_n_pol, logPow-logWeight & 0.491 & 0.449 \\
\hline 2 & TPSA, logPow-logWeight & 0.431 & 0.459 \\
\hline 1 & TPSA & 0.354 & 0.221 \\
\hline
\end{tabular}

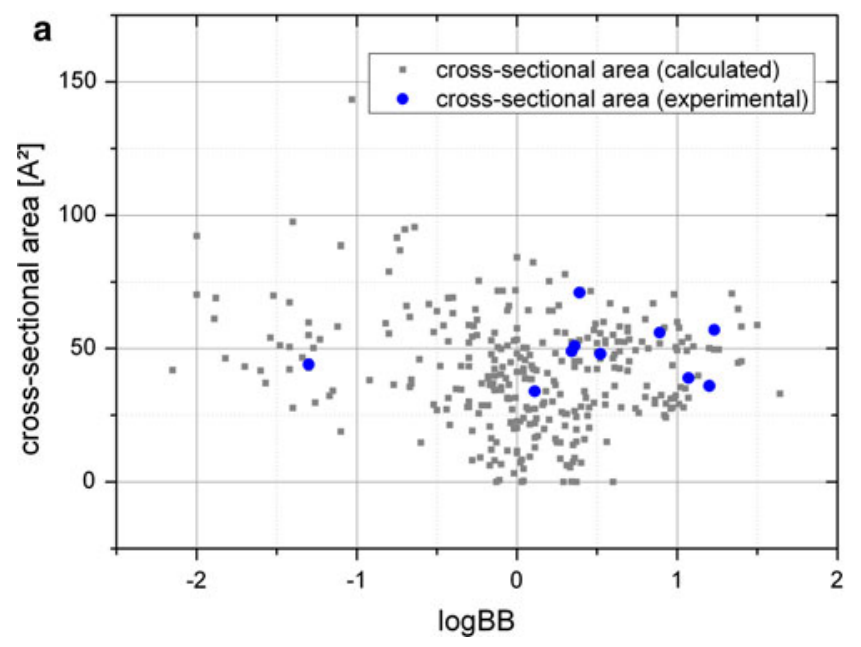

Fig. 4 a Experimental $\log B B$ plotted against 11 experimental and 362 calculated CSA show no correlation. Blue dots represent experimental CSA values, whereas grey dots are based on calculated CSA values. b Colour coded scatterplot of CSA versus $\log D$ (at

has also been found with the molecular weight. It was reported previously that molecular weight contributes to bioavailability in general [77]. Therefore we doubt that CSA provides more information with respect to $\mathrm{BBB}$ permeability than the number of atoms or molecular weight.

Number of descriptors, dataset size and accuracy

Whenever we tried to construct multiple linear regression models for $\log B \mathrm{~B}$ prediction on our large dataset, we failed to achieve results comparable to those reported by others using smaller data sets. To benchmark this relationship, linear regression models were built based on a single descriptor, but varying the composition of the training set.

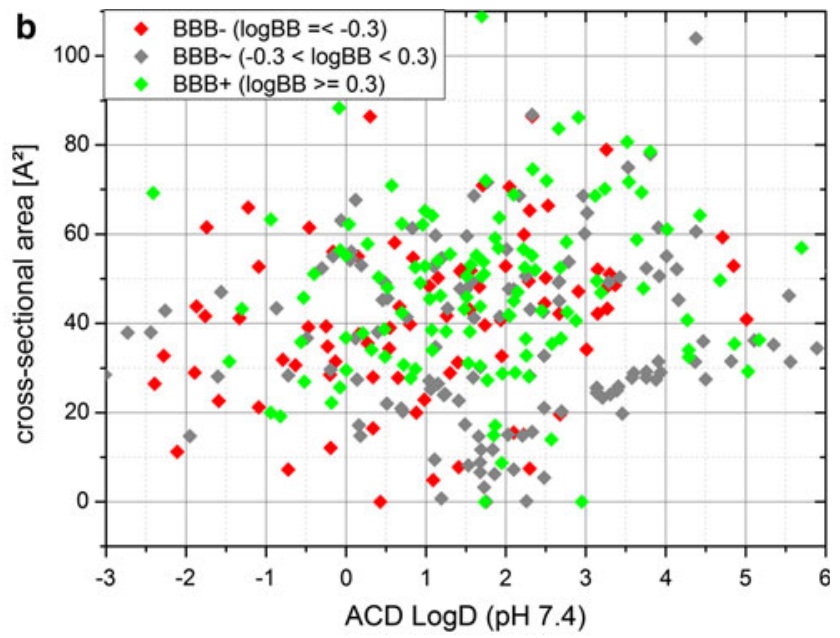

$\mathrm{pH}=7.4$ ), where green dots represent $\mathrm{BBB}$ permeable, red dots represent non-BBB permeable and gray dots represent unclassified compounds

As descriptor TPSA was chosen, since its impact on BBB permeability has not only been demonstrated by the models presented here but also by other researchers, for example by Kelder et al. [31]. In their study a set of 45 compounds was used to construct a regression model. Similarly, we constructed subsets from our dataset consisting of 50-350 compounds and calculated the squared correlation coefficient for each model. Each subset size was tested 500 times using different random seeds to cover different selection of compounds. Figure 6 illustrates that small sets show a large variability with respect to the squared correlation. Although the number of possible subsets is much lower for the large subsets, those are less likely to suffer from arbitrary correlations. This underlines the need for large datasets like the one we present here. 
Table 3 Various commonly-known descriptors correlate well with the CSA

\begin{tabular}{ll}
\hline Descriptor & Correlation coefficient \\
\hline a_count & 0.959 \\
b_count & 0.957 \\
apol & 0.955 \\
a_heavy & 0.929 \\
Weight & 0.897 \\
WeinerPath & 0.877 \\
mpc & 0.710 \\
n_pol & 0.645 \\
TPSA & 0.590 \\
I3 & 0.317 \\
n_PI & 0.264
\end{tabular}

CSA may therefore be regarded as an expensive replacement for much simpler descriptors. All descriptors are either standard MOE descriptors or explained in Table 1

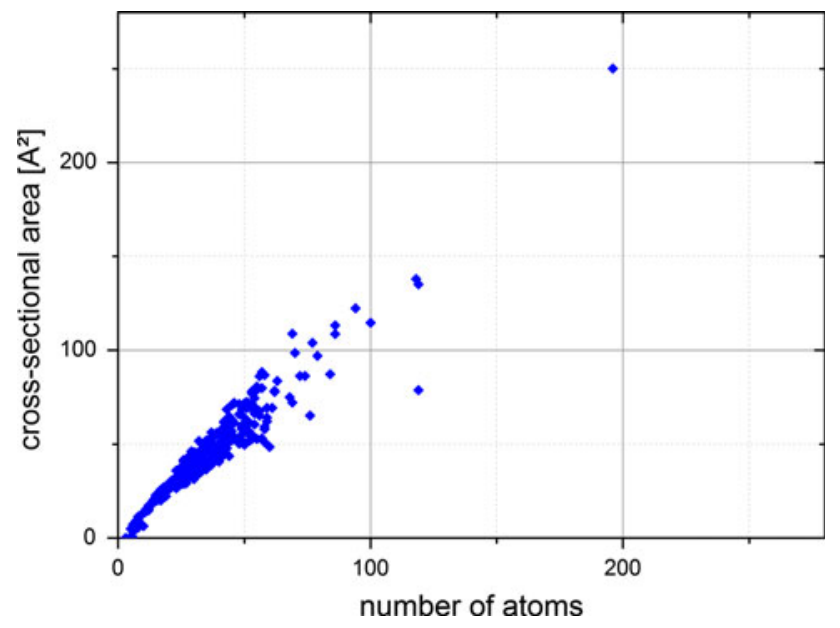

Fig. 5 CSA plotted against the number of atoms (a_count) reveals a remarkably high correlation $(r=0.959)$

\section{Qualitative models}

Focusing on a small number of descriptors, we were not able to obtain simple models with high performance using quantitative techniques. Thus we also calculated qualitative models to predict BBB permeability using a random forest. Again, we could compare our results to various published studies $[28,38,56]$.

For the qualitative models we used the same dataset as for the quantitative models, but converted $\log B \mathrm{~B}$ values into three bins. Compounds with a $\log B \mathrm{~B} \geq 0.3$ comprise the set of $\mathrm{BBB}$ permeable compounds, whereas compounds with a $\log \mathrm{BB} \leq 0.3$ are considered as not $\mathrm{BBB}$ permeable. The remaining compounds are excluded from the qualitative modelling. This left us with a set of 202 compounds

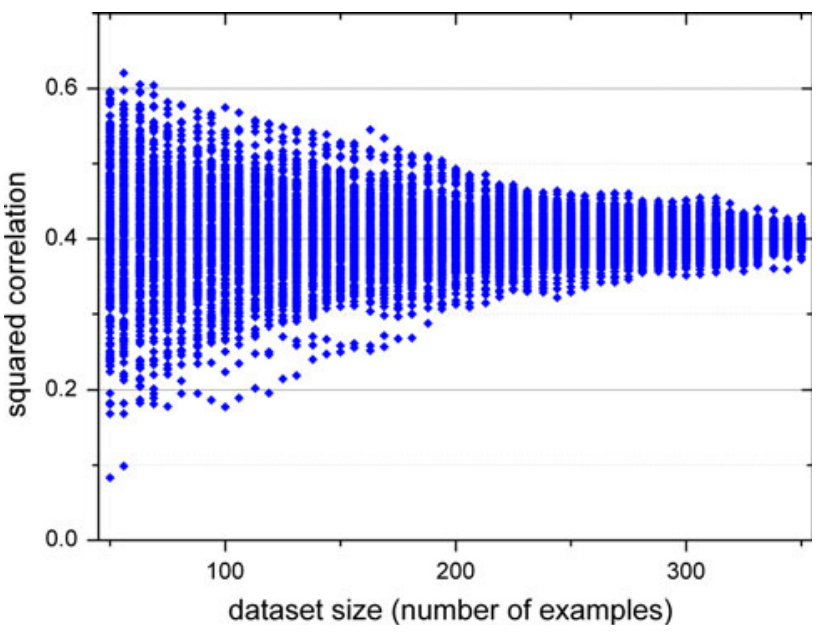

Fig. 6 Different training sets with 50-350 examples all selected from our dataset $(n=362)$ show that the size of the training set highly influences the performance given by squared correlation, even when constructed with exactly the same descriptor (TPSA) and the same procedure

Table 4 Accuracies (raw and validated) of the best models and prediction systems with up to 4 descriptors constructed using a beam feature search in combination with random forest learners

\begin{tabular}{llll}
\hline $\mathrm{n}_{\text {atts }}$ & Descriptor names & Accuracy $y_{\text {trees }=5}$ & $\begin{array}{l}\text { Accuracy } \\
\mathrm{n}=100\end{array}$ \\
\hline 4 & $\begin{array}{c}\text { TPSA, I3, QSUMN, } \\
\text { QSUMN/Weight }\end{array}$ & 1.000 & 0.878 \\
3 & $\begin{array}{l}\text { TPSA, QSUMN, QSUMN/ } \\
\text { Weight }\end{array}$ & 0.985 & 0.868 \\
2 & TPSA, QSUMN & 0.970 & 0.864 \\
1 & TPSA & 0.926 & 0.834 \\
\hline
\end{tabular}

for the training set. From the initial set of 886 descriptors, only 72 descriptors remained after preselection. Similar to the quantitative approach we aimed to obtain simple and interpretable models with a maximum of 4 concurrent descriptors. The beam search returned one model without misclassification (accuracy $=1.00$ ) using four descriptors. To evaluate the robustness of this model a bootstrap validation $(\mathrm{n}=100)$ was applied. The complete results are shown in Table 4.

The random forest prediction system based on four descriptors also achieves a high validated accuracy. The selected descriptors are similar to those obtained by multiple linear regressions and therefore highlight the importance of the following basic molecular properties:

- TPSA was selected in all models.

- QSUMN is the sum of charges on nitrogen atoms. This classifies all compounds by their charge on nitrogen atoms and subsequently also discriminates compounds having no nitrogen atom at all. 
Table 5 Intercorrelation matrix for the four descriptors used in the best random forest prediction model

\begin{tabular}{lrrrl}
\hline & TPSA & I3 & QSUMN & QSUMN/Weight \\
\hline TPSA & 1.000 & & & \\
I3 & 0.054 & 1.000 & & \\
QSUMN & -0.617 & -0.492 & 1.000 & \\
QSUMN/Weight & -0.374 & -0.536 & 0.790 & 1.000 \\
\hline
\end{tabular}

- QSUMN/Weight represents a size-intensive descriptor calculated from QSUMN and the molecular weight. For large compounds the molecular weight is the dominating factor for this descriptor.

- I3: Is -1 for acid compounds, +1 for basic compounds and 0 for the remaining compounds.

To analyse the dependence of the four descriptors we also calculated the intercorrelation matrix (Table 5). Although QSUMN and QSUMN/Weight are highly correlated, both seem be predictive for $\log B$ B. Especially compounds with higher molecular weight differ considerably for the two descriptors.

Table 6 compares the results from our calculations with results from other publications. The results clearly show that random forest prediction systems are well-suited to classify BBB permeability. Altogether, we outperformed many other models trained on datasets with similar sizes in terms of validated accuracy, even using fewer molecular properties.

In contrast to results from quantitative models, qualitative classification models are able to predict BBB permeability with high accuracy, especially when aiming for simple models based on a small number of descriptors. To quantify BBB permeability a more sophisticated and complex model is needed. However, we have shown that the number of descriptors that can be used is limited when looking at validated performances. Using a high number of descriptors for small datasets bears the risk of overfitting and arbitrary correlations.

In contrast, we focused on a simple prediction system that links BBB permeability to easily understandable molecular properties. Focusing on a small number of descriptors it might be easier to construct a binary classifier than to quantitatively predict BBB permeability.
Strengths and limitations

In the present study there are several novel findings:

- In addition to well-known descriptors, we added a significant number of descriptors that have never been evaluated and validated in the context of BBB prediction, for example size intensive descriptors (explained in [75]), and other novel descriptors listed in Table 1. Furthermore, we addressed the CSA which has been proposed as being predictive for BBB permeability. The qualitative models as shown in Table 4 include, in addition to TPSA, two of these novel descriptors.

- All prediction systems are limited by the experimental error of the data they are based on. Therefore, our set consists of compounds with experimental $\log B \mathrm{~B}$ values only, compiled from various publications.

- We developed an unparalleled compact and highlypredictive qualitative model validated by bootstrapping, that might act as general guideline for estimating BBB permeability.

\section{Conclusion}

In this work, we applied qualitative and quantitative in silico techniques to predict BBB permeability. For this purpose we created a reasonably large dataset $(n=362)$ of experimental $\log B \mathrm{~B}$ values. For each compound of the training set we calculated a broad set of descriptors ranging from simple atom count descriptors to computational more expensive descriptors like the CSA perdendicular to the amphiphilic axis. For this special descriptor were also able to validate calculated CSA with a set of experimentally measured values $(\mathrm{n}=32)$.

The best quantitative prediction system based on multiple linear regression without overfitting yielded a bootstrapped squared correlation coefficient of 0.521 . Qualitative models based on a random forest performed remarkably better. The best prediction system based on only four descriptors achieved a bootstrap validated accuracy of $88 \%$ (unvalidated 100\%). Remarkably, the CSA was not chosen by the feature selection algorithm used to select the most predictive descriptors. In contrast, a
Table 6 Results of classification systems for BBB permeability taken from the literature

SVM support vector machine, $D T$ decision tree, $R F$ random forest, $C V$ cross-validation, $B S$ bootstrapping

\begin{tabular}{llcclc}
\hline Method & Validation & Number of descriptors & Dataset size & Overall accuracy & Reference \\
\hline SVM & 10-fold CV & $<100$ & 351 & 83.0 & {$[28]$} \\
DT & - & 2 & 43 & 86.0 & {$[56]$} \\
SVM & 10-fold CV & 8 & 351 & 80.0 & {$[28]$} \\
SVM & 5-fold CV & 5 & 415 & 79.1 & {$[38]$} \\
RF & 100 BS & 4 & 202 & 88.2 & This work \\
\hline
\end{tabular}


combination of simple and well-known descriptors was found to be most useful to predict $\log B$.

Finally, we also showed that large and carefully comprised datasets, like the one presented here, reduce the risk of arbitrary correlations and result in more reproducible and robust models.

\section{Support information}

The SVL script to calculate and visualize the CSA perpendicular to the amphiphilic axis is provided as well as a spreadsheet file containing the whole set of compounds together with their corresponding $\operatorname{logBB}$ as well as a complete list of the descriptors calculated by ACD/Labs 10.0 and MOE 2010.10 for free download.

Acknowledgments This work was supported by the German Ministry of Education and Research (BMBF Grant No. 01EX1015B).

Open Access This article is distributed under the terms of the Creative Commons Attribution Noncommercial License which permits any noncommercial use, distribution, and reproduction in any medium, provided the original author(s) and source are credited.

\section{References}

1. Bradbury MWB (1993) The blood-brain barrier. Exp Physiol 78(4):453-472

2. Wager TT, Chandrasekaran RY, Hou X, Troutman MD, Verhoest PR, Villalobos A, Will Y (2010) Defining desirable central nervous system drug space through the alignment of molecular properties, in vitro ADME, and safety attributes. ACS Chem Neurosci 1(6):420-434. doi:10.1021/cn100007x

3. Nielsen PA, Andersson O, Hansen SH, Simonsen KB, Andersson G (2011) Models for predicting blood-brain barrier permeation. Drug Discov Today 16(11-12):472-475

4. Lacombe O, Guyol AC, Videau O, Pruvost A, Bolze S, Prevost C, Mabondzo A (2010) Brain penetration predictivity using in vitro primary rat and human cell-based blood-brain barrier models for drug discovery and development. Fundam Clin Pharmacol 24:8

5. Muster W, Breidenbach A, Fischer H, Kirchner S, Müller L, Pähler A (2008) Computational toxicology in drug development. Drug Discov Today 13(7-8):303-310

6. Pardridge WM (2007) Blood-brain barrier delivery. Drug Discov Today 12(1-2):54-61

7. Stenehjem DD, Hartz AM, Bauer B, Anderson GW (2009) Novel and emerging strategies in drug delivery for overcoming the blood brain barrier. Futur Med Chem 1(9):1623-1641. doi: 10.4155/fmc.09.137

8. Popescu BO (2009) Pathological changes of the blood-brain barrier in dementias. J Neurol Sci 283(1-2):250

9. Banks WA (2008) Developing drugs that can cross the bloodbrain barrier: applications to Alzheimer's disease. BMC Neurosci 9(Suppl 3):S2

10. HEd V, Kuiper J, AGd B, Berkel TJCV, Breimer DD (1997) The blood-brain barrier in neuroinflammatory diseases. Pharmacol Rev 49(2):143-156
11. Jong A, Huang S-H (2005) Blood-brain barrier drug discovery for central nervous system infections. Curr Drug Targ Infect Disord 5:65-72

12. Bickel U (2005) How to measure drug transport across the bloodbrain barrier. NeuroRX 2(1):15-26

13. Dehouck M-P, Méresse S, Delorme P, Fruchart J-C, Cecchelli R (1990) An easier, reproducible, and mass-production method to study the blood-brain barrier in vitro. J Neurochem 54(5): 1798-1801. doi:10.1111/j.1471-4159.1990.tb01236.x

14. Fricker G (2008) In vitro models to study blood-brain barrier function. In: Ehrhardt C, Kim KJ (ed) Drug absorption studies: in situ, in vitro and in silico models, vol VII. Biotechnology: pharmaceutical aspects. Springer, US, pp 397-417. doi:10.1007/ 978-0-387-74901-3_17

15. Lacombe O, Videau O, Chevillon D, Guyot A-C, Contreras C, Blondel S, Nicolas L, Ghettas A, Benech H, Thevenot E, Pruvost A, Bolze S, Krzaczkowski L, Prevost C, As M (2011) In vitro primary human and animal cell-based blood-brain barrier models as a screening tool in drug discovery. Mol Pharm 8(3):651-663. doi: $10.1021 / \mathrm{mp} 1004614$

16. Terasaki T, Hosoya K-I (2001) Conditionally immortalized cell lines as a new in vitro model for the study of barrier functions. Biol Pharm Bull 24(2):111-118

17. Boje KMK (2001) In vivo measurement of blood-brain barrier permeability. In: Current protocols in neuroscience. Wiley. doi: 10.1002/0471142301.ns0719s15

18. Di L, Kerns EH, Bezar IF, Petusky SL, Huang YP (2009) Comparison of blood-brain barrier permeability assays: in situ brain perfusion, MDR1-MDCKII and PAMPA-BBB. J Pharm Sci 98(6):1980-1991

19. Mensch J, LJ L, Sanderson W, Melis A, Mackie C, Verreck G, Brewster ME, Augustijns P (2010) Application of PAMPAmodels to predict $\mathrm{BBB}$ permeability including efflux ratio, plasma protein binding and physicochemical parameters. Int $\mathrm{J}$ Pharm 395(1-2):182-197

20. Reichel A, Begley DJ (1998) Potential of immobilized artificial membranes for predicting drug penetration across the blood-brain barrier. Pharm Res 15(8):1270-1274

21. Adenot M, Lahana R (2004) Blood-brain barrier permeation models: discriminating between potential CNS and non-CNS drugs including P-glycoprotein substrates. J Chem Inf Comput Sci 44(1):239-248

22. de Boer AG, van der Sandt ICJ, Gaillard PJ (2003) The role of drug transporters at the blood-brain barrier. Annu Rev Pharmacol Toxicol 43:629-656

23. de Lange ECM (2004) Potential role of ABC transporters as a detoxification system at the blood-CSF barrier. Adv Drug Deliv Rev 56(12):1793-1809

24. Miller DS (2010) Regulation of P-glycoprotein and other ABC drug transporters at the blood-brain barrier. Trends Pharmacol Sci 31(6):246-254

25. Bauer B, Hartz AMS, Fricker G, Miller DS (2005) Modulation of p-glycoprotein transport function at the blood-brain barrier. Exp Biol Med 230(2):118-127

26. Schinkel AH (1999) P-Glycoprotein, a gatekeeper in the bloodbrain barrier. Adv Drug Deliv Rev 36(2-3):179-194

27. Gratton JA, Abraham MH, Bradbury MW, Chadha HS (1997) Molecular factors influencing drug transfer across the blood-brain barrier. J Pharm Pharmacol 49(12):1211-1216

28. Kortagere S, Chekmarev D, Welsh WJ, Ekins S (2008) New predictive models for blood-brain barrier permeability of druglike molecules. Pharm Res 25(8):1836-1845

29. Hutter MC (2003) Prediction of blood-brain barrier permeation using quantum chemically derived information. J Comput Aided Mol Des 17(7):415-433 
30. Feher M, Sourial E, Schmidt JM (2000) A simple model for the prediction of blood-brain partitioning. Int J Pharm 201(2): 239-247

31. Kelder J, Grootenhuis PDJ, Bayada DM, Delbressine LPC, Ploemen J-P (1999) Polar molecular surface as a dominating determinant for oral absorption and brain penetration of drugs. Pharm Res 16(10):1514-1519

32. Abraham MH, Chadha HS, Mitchell RC (1994) Hydrogen bonding. 33. Factors that influence the distribution of solutes between blood and brain. J Pharm Sci 83(9):1257-1268

33. Mente SR, Lombardo F (2005) A recursive-partitioning model for blood-brain barrier permeation. J Comput Aided Mol Des 19(7): 465-481

34. Bendels S, Kansy M, Wagner B, Huwyler J (2008) In silico prediction of brain and CSF permeation of small molecules using PLS regression models. Eur J Med Chem 43(8):1581-1592

35. Norinder U, Haeberlein M (2002) Computational approaches to the prediction of the blood-brain distribution. Adv Drug Deliv Rev 54(3):291-313

36. Luco JM (1999) Prediction of the brain - blood distribution of a large set of drugs from structurally derived descriptors using partial least-squares (PLS) modeling. J Chem Inf Comput Sci 39(2):396-404. doi:10.1021/ci980411n

37. Crivori P, Cruciani G, Carrupt PA, Testa B (2000) Predicting blood-brain barrier permeation from three-dimensional molecular structure. J Med Chem 43(11):2204-2216

38. Li H, Yap CW, Ung CY, Xue Y, Cao ZW, Chen YZ (2005) Effect of selection of molecular descriptors on the prediction of blood-brain barrier penetrating and nonpenetrating agents by statistical learning methods. J Chem Inf Model 45(5):1376-1384. doi:10.1021/ci050135u

39. Wang Z, Yan A, Yuan Q (2009) Classification of blood-brain barrier permeation by Kohonen's self-organizing neural network (KohNN) and support vector machine (SVM). QSAR Comb Sci 28(9):989-994

40. Zhang L, Zhu H, Oprea TI, Golbraikh A, Tropsha A (2008) QSAR modeling of the blood-brain barrier permeability for diverse organic compounds. Pharm Res 25(8):1902-1914

41. Hemmateenejad B, Miri R, Safarpour MA, Mehdipour AR (2006) Accurate prediction of the blood-brain partitioning of a large set of solutes using ab initio calculations and genetic neural network modeling. J Comput Chem 27(11):1125-1135

42. Teixido M, Belda I, Rosello X, Gonzalez S, Fabre M, Llora X, Bacardit J, Garrell JM, Vilaro S, Albericio F, Giralt E (2003) Development of a genetic algorithm to design and identify peptides that can cross the blood-brain barrier 1. Design and validation in silico. QSAR Comb Sci 22(7):745-753. doi: 10.1002/qsar.200320004

43. Narayanan R, Gunturi SB (2005) In silico ADME modelling: prediction models for blood-brain barrier permeation using a systematic variable selection method. Bioorgan Med Chem 13(8):3017-3028. doi:10.1016/j.bmc.2005.01.061

44. Vastag M, Keserü GM (2009) Current in vitro and in silico models of blood-brain barrier penetration: a practical view. Curr Opin Drug Discov Dev 12(1):115-124

45. Gleeson MP (2008) Generation of a set of simple, interpretable ADMET rules of thumb. J Med Chem 51(4):817-834. doi: $10.1021 / j m 701122 \mathrm{q}$

46. Pardridge WM (2003) Blood-brain barrier drug targeting: the future of brain drug developement. Mol Interv 3(2):90-105. doi: 10.1124/mi.3.2.90

47. Liu R, Sun H, So S-S (2001) Development of quantitative structure - property relationship models for early ADME evaluation in drug discovery. 2. Blood-brain barrier penetration. J Chem Inf Comput Sci 41(6):1623-1632. doi:10.1021/ci010290i
48. Waring MJ (2009) Defining optimum lipophilicity and molecular weight ranges for drug candidates-Molecular weight dependent lower $\log \mathrm{D}$ limits based on permeability. Bioorg Med Chem Lett 19(10):2844-2851

49. Liu X, Testa B, Fahr A (2011) Lipophilicity and its relationship with passive drug permeation. Pharm Res 28(5):962-977. doi: 10.1007/s11095-010-0303-7

50. Kramer C, Beck B, Clark T (2010) A surface-integral model for log POW. J Chem Inf Model 50(3):429-436. doi:10.1021/ci900431f

51. Muehlbacher M, Kerdawy AE, Kramer C, Hudson B, Clark T (2011) Conformation-dependent QSPR models: $\log P O W$. J Chem Inf Model 51(9):2408-2416. doi:10.1021/ci200276v

52. Abraham MH (2010) The permeation of neutral molecules, ions, and ionic species through membranes: brain permeation as an example. J Pharm Sci 100(5):1690-1701. doi:10.1002/jps.22404

53. Fischer H, Gottschlich R, Seelig A (1998) Blood-brain barrier permeation: molecular parameters governing passive diffusion. J Membr Biol 165(3):201-211

54. Keseru GM, Molnar L (2000) High-throughput prediction of blood - brain partitioning: a thermodynamic approach. J Chem Inf Comput Sci 41(1):120-128. doi:10.1021/ci000043z

55. Seelig A (2007) The role of size and charge for blood-brain barrier permeation of drugs and fatty acids. J Mol Neurosci 33(1):32-41

56. Gerebtzoff G, Seelig A (2006) In silico prediction of blood-brain barrier permeation using the calculated molecular cross-sectional area as main parameter. J Chem Inf Model 46(6):2638-2650. doi: 10.1021/Ci0600814

57. Hughes JD, Blagg J, Price DA, Bailey S, DeCrescenzo GA, Devraj RV, Ellsworth E, Fobian YM, Gibbs ME, Gilles RW, Greene N, Huang E, Krieger-Burke T, Loesel J, Wager T, Whiteley L, Zhang Y (2008) Physiochemical drug properties associated with in vivo toxicological outcomes. Bioorg Med Chem Lett 18(17):4872-4875

58. Ertl P, Rohde B, Selzer P (2000) Fast calculation of molecular polar surface area as a sum of fragment-based contributions and its application to the prediction of drug transport properties. J Med Chem 43(20):3714-3717

59. Labute P (2000) A widely applicable set of descriptors. J Mol Graph Model 18(4-5):464-477

60. Molecular Operating Environment MOE 2010.10 (2010)

61. Li Q, Cheng T, Wang Y, Bryant SH (2010) PubChem as a public resource for drug discovery. Drug Discov Today 15(23-24): 1052-1057

62. Lipinski CA, Lombardo F, Dominy BW, Feeney PJ (2001) Experimental and computational approaches to estimate solubility and permeability in drug discovery and development settings. Adv Drug Deliv Rev 46(1-3):3-26

63. Vilar S, Chakrabarti M, Costanzi S (2010) Prediction of passive blood-brain partitioning: straightforward and effective classification models based on in silico derived physicochemical descriptors. J Mol Graph Model 28(8):899-903

64. Platts JA, Abraham MH, Zhao YH, Hersey A, Ijaz L, Butina D (2001) Correlation and prediction of a large blood-brain distribution data set—an LFER study. Eur J Med Chem 36(9):719-730

65. Abraham MH, Ibrahim A, Zhao Y, Acree WE (2006) A data base for partition of volatile organic compounds and drugs from blood/ plasma/serum to brain, and an LFER analysis of the data. J Pharm Sci 95(10):2091-2100

66. Garg P, Verma J (2006) In silico prediction of blood brain barrier permeability: an artificial neural network model. J Chem Inf Model 46(1):289-297

67. Guerra A, Páez JA, Campillo NE (2008) Artificial neural networks in ADMET modeling: prediction of blood-brain barrier permeation. QSAR Comb Sci 27(5):586-594 
68. Rose K, Hall LH, Kier LB (2002) Modeling blood-brain barrier partitioning using the electrotopological state. J Chem Inf Comput Sci 42(3):651-666

69. Konovalov DA, Coomans D, Deconinck E, Vander Heyden Y (2007) Benchmarking of QSAR models for blood-brain barrier permeation. J Chem Inf Model 47(4):1648-1656

70. Zerara M, Brickmann J, Kretschmer R, Exner TE (2009) Parameterization of an empirical model for the prediction of n-octanol, alkane and cyclohexane/water as well as brain/blood partition coefficients. J Comput Aided Mol Des 23(2):105-111

71. Xue Y, Yap CW, Sun LZ, Cao ZW, Wang JF, Chen YZ (2004) Prediction of P-glycoprotein substrates by a support vector machine approach. J Chem Inf Comput Sci 44(4):1497-1505. doi:10.1021/ci049971e

72. Wang Y-H, Li Y, Yang S-L, Yang L (2005) Classification of substrates and inhibitors of P-glycoprotein using unsupervised machine learning approach. J Chem Inf Model 45(3):750-757. doi:10.1021/ci050041k
73. Varma MVS, Sateesh K, Panchagnula R (2004) Functional role of P-glycoprotein in limiting intestinal absorption of drugs: contribution of passive permeability to P-glycoprotein mediated efflux transport. Mol Pharm 2(1):12-21. doi:10.1021/mp0499196

74. Advanced Chemistry Development Inc (2010) ACD/PhysChem. version 10.0 edn, Toronto

75. Purvis G (2008) Size-intensive descriptors. J Comput Aided Mol Des 22(6):461-468

76. Andres C, Hutter MC (2006) CNS permeability of drugs predicted by a decision tree. QSAR Comb Sci 25(4):305-309

77. Fu X-C, Wang G-P, Shan H-L, Liang W-Q, Gao J-Q (2008) Predicting blood-brain barrier penetration from molecular weight and number of polar atoms. Eur J Pharm Biopharm 70(2):462-466

78. Furcy D, Koenig S (2005) Limited discrepancy beam search. Paper presented at the Proceedings of the 19th international joint conference on Artificial intelligence, Edinburgh, Scotland 